\title{
INHALTSVERZEICHNIS
}

\section{A UFSATZE}

Altenhain, F. K., Ein Bootstrap-Integrator . . . . 234

Bauer, A., Untersuchung der Stabilitätsgrenze eines Nachlaufreglers mit Stabilisierung über ein Parallel-T-Filter

Bauer, M., Die Welligkeit der Gleichspannung von Tachometerdynamos und ihr Einflu $B$ auf die Regelung................ 203

Becker, H., Zur Theorie der Mehrfachregelung . . 133

- Das Umkehrverfahren und die Näherungslösungen nichtlinearer Differentialgleichungen . . . . . 168

Biederstedt, U., Der Magnettrommelspeicher . . . 81

Costake, N. N., Steuerung und Regelung der dieselelektrischen Eisenbahnfahrzeuge . . . . 230, 312

Engl, W., Zur Frage der ,wirksamen Fläche“ von Tellermembranen . . . . . . . . . . . . . 165

Euler, K., Regelsysteme mit Laufzeit . . . . . . . . . . 89

Gonnermann, H., Ein Verfahren zur Auflösung algebraischer Gleichungen 4. Grades . . . . . . .

Gumin, H., Prinzipien und Grundbausteine für digitales Rechnen . . . . . . . . . . . . . . .

Há j ek, J., Vereinfachte Stabilitätskriterien für lineare Regelsysteme . . . . . . . . . . . . .

Hannakam, L., Übergangsverhalten des DrehstromSchleifringläufers .......... . . 393, 421

Heller, G., Ein elektrisches Analogiegerät für Totzeiten 266

Hengstenberg, J., Meßtechnik und Automatik in der Chemie . . . . . . . . . . 338

Herschel, R., Ubber die Stabilität von Regelsystemen mit quadratischen Abhängigkeiten im PI-Regler. 240

Hofmann, R., Ein Verfahren zur numerischen Berechnung von Originalfunktionen bei gegebenen gebrochen rationalen Bildfunktionen .

-, Kriterium für das Vorhandensein von Mehrfachwurzeln oder einer ungeraden bzw. geraden Anzahl konjugiert komplexer Wurzelpaare algebraischer Gleichungen .... . . . . . . . . 310

Hutarew, G., Durchfluß und Verlusthöhe in Absperrorganen und Regelorganen . . . . . . . . . . 193

Jong, J. J. de, Wirtschaftliche Aspekte der Automatisierung in der Erdölindustrie. . . . . . . . . 383

Jötten, R., Zur Theorie und Praxis der Regelung von Stromrichterantrieben ......... . 5, 44

Junker, B., Automation in der Konservenindustrie . 389

Kehoe, T. J.s. Silva

Kind, H., Frequenzgänge mehrstufiger Magnetverstärker mit Rückführungen . . . . . 86

Kretzschmer, F., Die Bemessung von Regelventilen 351

Lichtblau, K., Die Darstellung von Regelungsvorgängen mittels zeitabhängiger Störungen . . . . 306

Lintz, H. s. E. Wintergerst

Lutz, K., Die Berechnung des Schwebekörper-Durchflußmessers 355

Marosky, F., Marktforschung für Regler in der Verfahrensindustrie. . . . . . . . . . . . . .

Mesarović, M., Die Bedeutung der höheren Ableitungen der Regelgröße in Regelkreisen mit Stoß-

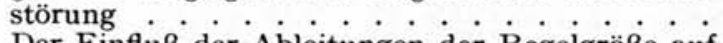

-, Der Einfluß der Ableitungen der Regelgröße auf den Regelkreis mit statistischer Störung . . . . 198
Noebels, H. J. s. Silva

Peterka, V., Eine einfache numerische Methode zur Berechnung der Übergangsprozesse in linearen Regelkreisen .............

Rechberger, H., Eine stetigähnliche Turbinenregelung fiir Kleinwasserkraftanlagen

Schließmann, H., Über die optimale Bemessung von Regelsystemen mit Laufzeit. . . . . . . . 272, 440

-, Über ein Verfahren zur optimalen Regelung von Systemen mit Totzeit . . . . . . . . . . . . 418

Schlitt, H., Zur Anwendung statistischer Verfahren in der Regelungstechnik . . . . . . . .

Schraivogel, R. A., Die Anwendung des NicholsDiagramms auf die Berechnung rationaler Funktionen . . . . . . . . . 206

Silva, M., N. J. Noebels und T. J. Kehoe, Analytische Behandlung der $p_{\mathrm{H}^{-}}$-Regelung . . . . . 118

Starkermann, R., Gegenseitige Beeinflussung der Regelgrößen in Mehrkreissystemen . . . . . 301

Strejc, V., Näherungsverfahren für aperiodische Übergangscharakteristiken . . . . . . . . . 124

Sturm, B., Die Technik der automatischen Energiekontrolle . . . . . . . . . . . . . . . . . . 378

Süss, R., Projektierung von $p_{\mathrm{H}}$-Regelanlagen . . . 344

Tscha uner, J., Die Stabilität eines besonderen Regelsystems . . . . . . . . . . . . . . . . . . 247

Wilmowsky, H. J. v., Über die selbsttätige laufende Messung von Blutdruckgrößen mit Hilfe einer Regeleinrichtung ........ . 427

Wintergerst, E., und $\mathrm{H}$. Lintz, Eigenschaften und Anwendungen von Metallmembranen . . . . . 160

Zypkin, Ja. S., Über die Beseitigung des Einflusses von Totzeit auf die dynamischen Eigenschaften von nichtlinearen Impulsregelsystemen . . 196, 440

\section{AUS DER INDUSTRIE}

Beckman Instruments $\mathrm{GmbH}$, München Kontinuierliche Messung des im Wasser gelösten Sauerstoffs . . . . . 253

Eckardt, J. C. - , Stuttgart Planung von Regelanlagen . . . . . . . . . . 18

Elektro Spezial GmbH, Hamburg Miniatur-Thermoelemente . . . . . . . . . 436

Heinlein, H. - Eßlingen Kontaktschutzrelais . . . . . . . . 22

Honeywell $\mathrm{GmbH}$, Frankfurt a. Main Electrik Tel-O-Set-System . . . . . . . . 213

Kent, Georg - Ltd., London Automatische Regelung eines Kessels mit Zyklon-

Kent-Barton feuerung . 210 Differenzdruckmesser . . . . . . . . . . 174

Novotechnik KG, Stuttgart Analogrechner A 398 von Rochar-Electronique . 253

Peters, Claudius - AG, Hamburg Automatische Siloinhaltskontrolle . . . . . . 284 
Rifox Spezialarmaturen GmbH, Bremen Kondensatableiter .

Siemens \& Halske AG, München

Analog-Digital-Umsetzer mit Transistoren

Siemens \& Halske, Wernerwerk für Meßtechnik,

Karlsruhe

Elektropneumatischer Regler des Teleperm-Telepneu-Regelsystems

Meß- und Regelanlage am Versuchskonverter der Phoenix-Rheinrohr . . . . . . . . 248

Automatisierungsaufgaben und deren Lösung durch verschiedene Steuersysteme . . . . . 317

Das Telepneu-Multipliziergerät in pneumatischen Einheitsregelsystemen . . . . . . . 433

Siemens-Schuckert AG, Erlangen

Nachbildung eines Spaltreaktors . . . . . . . 21

Reaktor in München . . . . . . . . . . . 58

Sunvic Regler $\mathrm{GmbH}$, Solingen

Präzisionsgeräte für die Tankmessung . . . . . 56

\section{GRUNDLAGEN}

Henning, H., Der Blockschaltplan in der Regelungstechnik

\section{MITTEILUNGEN}

\section{ALLGEMEINES}

\section{Fa. Kent, Niederlassung in Deutschland}

Erwiderung zu ,Rückblick auf die Tagung Steuerungen und Regelungen in der Technik elektrischer Antriebe der VDI/VDE-Fachgruppe Regelungs-

Telefunken-Stammkapital auf 100 Millionen erhöht. . 135

2. Amerikareise zum Studium der Regelungstechnik und des Einsatzes elektronischer Geräte . . . . . . 180

Archiv wissenschaftlicher Schirften . . . . . . . . 213

Zusammenarbeit von Kali-Chemie AG, Hannover, und G. Bischoff KG, Essen . . . . . . . .

Eröffnung des ersten technischen Büros der ElektroSpezial GmbH . . . . . . . . 256

Aufwärtssteigende Entwicklung in der elektrischen Meßtechnik

Unterteilung der Klasse $\dot{\mathrm{G}} 05^{\circ}$, , Regeln und Steuern der internationalen Klassifikation für Erfinderpatente . . . . . . . . . . 321

Produktionserweiterung der Contraves AG . . . . . 323

Richtfest bei Telefunken in Heilbronn . . . . . . 360

VDI/VDE-Richtlinien über Benennung auf dem Gebiet der Flugregelung

Neue Geschäftsführung der Deutschen Worthington $\mathrm{GmbH}$. . . . . . . . . . . . 362

Pipeline Lavera-Strasbourg-Karlsruhe . . . 389

Wie ordnet und findet man regelungstechnische $\mathrm{Pa}$ tentschriften und Literatur? . . . . . . . . 438

\section{BERICHTE ÜBER TAGUNGEN}

Tagung ,,Steuerungen und Regelungen in der Technik elektrischer Antriebe" in Essen . . . . . .

4. Tagung der Schweizerischen Gesellschaft für Automatik in Zürich .

1.Internationaler IFAC-Kongre $B$ in Moskau 1960.

20.NAMUR-Hauptsitzung in Frankfurt . . . . . . 99

Congresso International de Automatica in Madrid. . 103

Fachbuchschau deutscher Verleger auf der Deutschen Industriemesse Hannover 1959

GAMM-Jahrestagung 1959 .

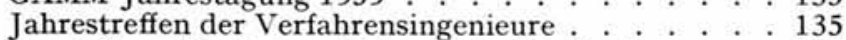

Ausstellung für Automatisierung in England 135

Österreichischer Arbeitsausschuß für Automatisierung 180

3. Kongre $B$ der Europäischen Vereinigung der Hersteller von Ausrüstungsgegenständen für die Mineralindustrie . . . . . . . . . 180

Tagung des VDI in Aachen $. \quad . \quad . \quad 254$

4. Tagung und Ausstellung für Automatisierung und Instrumentierung in Mailand ..... . . 254, 323
5. Tagung der Schweizerischen Gesellschaft für Automatik . . . . . . . . 25 Wintertagung der SGA in Basel . . . . . . . . 255

Jahrestreffen 1959 der Verfahrensingenieure : . . . 255 IV.Ausstellung mit Seminar der JUREMA . . . . 285 Vorträge der IFAC auf der ISA-Jahreskonferenz 1959. 285 Tagung der VDI/VDE-Fachgruppe Regelungstechnik. 285 $\mathrm{MeB}$ - und Regeltechnik in der Brennstoffwärme-Anwendung . . . . . 323

Generalversammlung der IFAC in Chicago . . . . 398

\section{PERSÖNLICHES}

Bukovics, E. . . . . . . . . . . 362

Hornauer, W. . . . . . . . . . 362

Leonhard, A . . . . . . . . . . . . . 437

Profos, P. . . . . . . . . 59

Schmidt, H. . . . . . . . . . . . . . 437

\section{SCHRIFTTUM}

AEG-Buch ,Wärmetechnische Regler und Regelanlagen" . . . . . . . . . . . . . 66

- "Energieübertragungen mit Höchstspannungen". 215

-, "Elektrische Anlagen in der Faserstoffindustrie". 288

-, "Motoren für Drehstrom und Wechselstrom" . . . 363

— Stromrichterantriebe. . . . . . . 400

Altrichter, E., Das Magnetband . . . . . . . . 136

Andronow, A. A., A. A. Witt und S. E. Chaikin, Theorie der Schwingungen ...... 363

Angerer, E. v., Technische Kunstgriffe bei physikalischen Untersuchungen . . . . . . . . . 324

Brötz, W., Grundriß der chemischen Reaktionstechnik .............. 286

Cahen und Treille, Abriß der Kernenergie. . . . . 67

Cald well, S. H., Switching circuits and logical design 30

Chaikin, S. E. s. Andronow

Chudobin, Automatische Regelung des radialen Schleifdrucks ..... . 399

Cuénod, M., und L. Pu n, Les fluctuations aléatoires et l'imprécision des réglages industriels . . . . . 103

Doetsch, G., Einführung in die Theorie und Anwendung der Lapalce-Transformation . . . . . . 216

Goldberg, S., Introduction to difference equations with illustrative examples from economics, psychology and sociology . . . . . . .

Grabbe, Ramo, Wooldridge, Handbook of Automation, Computation and Control . . . . . . 104

Gröbner, W., Matrizenrechnung . . . . . . . . . . . . . . 399

Hölzler, E., und H. Holzwarth, Theorie und Praxis der Pulsmodulation ......... 215

Holzwarth, H. s. Hölzler

Huber, R., Trockenbatterien.

Kagan, J. I., Automatische Messen von Wellendurchmessern während des Schleifens . . . . . . . .

K ühl, H., Probleme des Kreuzstrom-Wärmeaustauschers . . . . . . . . . . . . 438

Lurje, A. I., Einige nichtlineare Probleme aus der Theorie der selbsttätigen Regelung . . . . . 216

Moeller-Werr, Leitfaden der Elektrotechnik. . . . 324

Opitz, Untersuchungen von elektrischen Antrieben, Steuerungen und Regelungen an Werkzeugmaschinen .............. 400

Pun s. Cuénod

Recknagel-Sprenger, Taschenbuch für Heizung, Lüftung und Klimatechnik.

Rolf, E., Der Kontaktumformer . . . . . . . . . 286 
Romanow, W. A., Die erste automatische Taktstraße von Zahnrädern .. . . . . . . . . . . . 399

Sharow, N. T., Grundlagen der Automatisierung in der Gießerei

Solodownikow, W. W. Grundlagen der selbsttätigen Regelung . . . . . . . . . . 324, 363

Strutt, M. J. O., Elektronenröhren . . . . . . . 66

Tuttle, D. F., Network Synthesis .

Wahlenmayer, F., Selbsttätige Regelung von Klimaalagen

Witt, A. A. s. Andronow

Zemanek, H., Elementare Informationstheorie . . 136

Zurmühl, R., Praktische Mathematik für Ingenieure und Physiker . . . . . . . . . . . . . 256

Zypkin, Ja., S., Vorlesungen zur Regelungstheorie . 68

Nichtlineare Regelungsvorgänge. Beiheft zur Regelungstechnik

Physikalisches Taschenbuch . . 134

Regler und Regelungsverfahren der Nachrichtentechnik 214

Maschinen-Einkaufsführer 1959 . . . . . . . . . . 285

Automatik-Katalog 1959 . . . . . . . 323

Messen und Regeln in der chemischen Technik . . . 439

\section{SACHWORTVERZEICHNIS}

Algebraisch

Verfahren zur Auflösung -er Gleichungen vier-

Kriterium für bestimmte Wurzelpaare - er Gleichungen .............. 310

Analog-Digital-Umsetzer . . . . . . . . . . . . . 95

Analogrechner .. . . . . . . . . . . 371, 407

Bootstrop-Integrator ......... . . 234

- Rochar-Electronique . . . . . . . . . . 253

Elektrisches Analogiegerät für Totzeiten. . . . 266

Telepneu-Multipliziergerät . . . . . . . . . 433

Anzeigeregler . . . . . 261

Autoklavenregelanlage . . . . . . . . . . . . . . . 185

Automatisierung .. . . . . . . . . . . . . . . 18

— in der Gießerei . . 67

-saufgaben und deren Lösung durch verschiedene Steuersysteme .. . . . . . . 317

Automatik in der Chemie .... 338

— in der Erdölindustrie . . . . . . . . 383

- in der Konservenindustrie . . . . . . . . . . 389

Behälterstandsmeßgeber . . . . . . . . . . 332

Behälterstandsmessung . . . . . . . . . . . . . 340

Betriebskontrolle, Maschinelle Rechner in der - . . . 342

Blockschaltplan in der Regelungstechnik . . . . . . 60

Blutdruckgrößen, Laufende Messung von - . . . . . 427

Brennerüberwachung. .. . . . . . . . . . . . 409

Brennkammerluft, Regelung der - .

Brennstoffwärme-Anwendung, Meß- und Regeltechnik

in der - . . . . . . . . . . . . . . . . . 323

Bunkerstandanzeiger, Elektronischer - . . . . . . . 284

Chemie, Meßtechnik und Automatik in der - . . 338, 439

Chromatograph für Gasanalysen . . . . . . . . . 78

Dämmerungsschalter. . . . . . . . . . . . . 283

Dehnung, Messung und Regelung der - laufender

Bahnen ............. 178

Destilliergerät, Automatisches - . . . . . . . . 257

Dichtemesser . . . . . . . . . . . . . . . 376

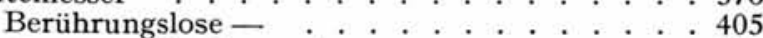

Dickenmesser, Berührungslose - . . . . . . . 405

Differenzdruck

Pneumatischer Meßwertumformer für - . . . . 74 digital

-messer ohne Quecksilber ........ . 174

Prinzipien und Grundbausteine für —es Rechnen. 41

- er Spannungsschreiber . 73

-Rechner RW 300 . . . . . . . . . . . . . 334
Seite

Elektronisches - -Voltmeter . . . . . . . 370

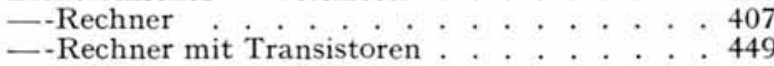

Dosierschwimmer, Universal- _ . . . . . 57

Drehspulmeßrelais . . . . . . . . . . . 38

Drehstrom-Schleifringläufer, Übergangsverhalten des

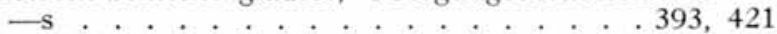

Drehzahlfeinmeßgerät . . . . . . . . . . . . . . . . 142

Drehzahlmesser, Berührungsloser - . . . . . . . 371

Druckmeßgerät ......... 167

Druckmessung . . . . . . . . . . . . . . . . . 341

Elektronische - . . . . . . . . . . . . . 225

Druckreduzierstationen, Regelung von -. . . . . . 301

Druckregler, Pneumatischer - 33

DurchfluB in Absperr- und Regelorganen . . . . . . 193

Durchflußmengenmesser . . . . . . . . . . . 445

Durchflußmesser $\ldots ., 293$

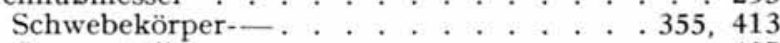

Ganzmetall-_. . . . . . . . . . . . . . . . 407

Durchflußtransmitter . . . . . . . . . . . . 379

Eisenbahnfahrzeuge, Steuerung und Regelung diesel-

lektrischer - 230, 312

elektrisch s. Einzelbegriffe

elektronisch s. Einzelbegriffe

Energiekontrolle, Automatische -. . . . . . . . . 378

Energieversorgung, Regelung in der -. . . . . . . 337

Erdölindustrie, Automatisierung in der - . . . . . 383

Fernanzeige mit Langskaleninstrument. . . . . . 258

Feuchteregler. .. . . . . . . . . . . . 110

Flammenwächter . . . . . 227

Flugregelung, Benennung auf dem Gebiet der - . . 360

Fotowiderstände .. . . . . . . . . . . . . 335

Fotozellen . . . . . . . . . . . . . . . . . 335

Frequenzgänge mehrstufiger Magnetverstärker . . . 86

Geber s. Einzelbegriffe

Gleichspannungsquellen, Elektronisch stabilisierte - 412

Halbleiter-Bauelemente, Einheitliche Bezeichnung für

一. . . . . . 369

Heizungsregler .. . . . . . . 329, 413

Hubgerät, Elektrohydraulisches - . . . . . . . 38

IFAC

1. Internationaler - Kongre $B$ in Moskau. . . . 60

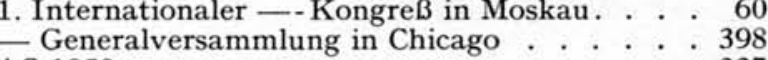

ILMAC 1959 . . . . . . . 337

Impulsregelsysteme, Beseitigung des Einflusses von

Totzeit in nichtlinearen $-\mathrm{n}$. . . . . . . . . 196

Industriemesse Hannover. . . . . . . .

Informationstheorie, Elementare - . . . . . . . . 136

Integrator s. Analogrechner

Kaltkathodenröhren, Wirkungsweise und Anwendung

von - . . . 280

Kaskadenregelung . Kessel

Automatische Messung und Auswertung bei -

versuchen ........... . 179

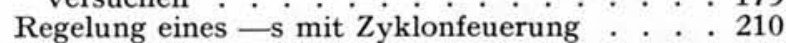

Klassiergerät, Elektronisches - mit Meßwertspeicher 259

Kleinformregler . . . . . . . . . . . . . . . 19

Kleinstmotor, Gleichstrom-- . . . . . . . . . . 263

Klimaanlagen, Regelung von - . . . . . . . . . 135

Kompensationsschreiber, Elektronischer - . . . . . 148

Kondensatableiter. . . . . . . . . . . 150, 175

Konservenindustrie, Automation in der - . . . . 389

Konstantspannungsgeneratoren . . . . . . . . . . 190

Kontaktumformer. . . . . . . . . . . . . . . . . 286

Konverter, Regelanlage an Versuchs-. . . . . . . . 248

Konzentrationsmessung . . . . . . . . . . . . . 341

Kraftmessung, Elektronische - . . . . . . . . . . 225

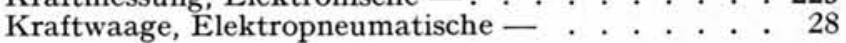

Laplace-Transformation

Theorie und Anwendung der - . . . . . . . 216

Numerische Berechnung von Originalfunktionen

beigegebenen gebrochen rationalen Bildfunktionen 269 
Laufzeit s. Totzeit

Lichtsteuerschaltung. . . . . . . . . . . . . . . 282

Logistat-Systeme . . . . . . . . . . . . . . . . 368

Magnetkernrelais . . . . . . . . . . . . . . 333

Magnettrommel-Speicher . . . . . . . . . 81

Magnetventil ............ 110

Magnetverstärker, Frequenzgänge mehrstufiger mit Rückführungen . . . . . . . . . . . . 86

Matrizenrechnung . . . . . . . 399

Mehrfachregelung, Theorie der . . . . . . 133

Mehrkreissysteme, Gegenseitige Beeinflussung der Regelgrößen in $-\mathrm{n}$. . . . . . . . . . . . 301

Membran

Eigenschaften und Anwendung von Metall-n. . 160

„Wirksame Fläche“ von Teller-en . . . . . . 165

Mengenmessung. . 340 Geräte zur - . . . . . . . . . . . . . . 115

Meßumformer s. Einzelbegriffe

Meßverstärker ..... 331

Meßwertgeber, Mechanisch-elektrischer — . . . . 146

Meßwertüberwachungssysteme . . . . . . . . . 318

Nachlaufregler, Stabilitätsgrenze eines $-\mathbf{s}$. . . . . 93

NAMUR, 20. - -Hauptsitzung . . . . . . . . . . 99

Nichols-Diagramm zur Berechnung rationaler Funktion . . . . . . . . . . 206 nichtlinear

ce Regelvorgänge. . . . . . . . . . . . . 67

Näherungslösungen —er Differentialgleichungen . 168

Totzeit in —en Impulsregelsystemen. . . . . . 196 Einige - e Probleme der Regelungstechnik . . . 216

Quadratische Abhängigkeiten im PI-Regler. . . 240

Niveauregelung . . . . . . . . . . . . 391, 451

Nullindikator, Oszillografischer - . . . . . . . 147

optimal

Einfluß der Ableitungen auf die $-\mathrm{e}$ Einstellung des Reglers . . . . . . . . . . . . . .

- Bemessung von Regelsystemen mit Lauf-

zeit ................272, 418

Optimierung von Regelvorgängen bei statistischer

Störung . . . . . . . . . . . . . 201

Patentklassifikaton, Unterteilung der Klasse G 05 "Regeln und Steuern" . . . . . . . . . . . . 321

$p_{\mathrm{H}}$-Meßgerät . . . . . . . . . . . . . . 143

$p_{\mathrm{H}}$-Regelung

Analytische Behandlung der - . . . . . . . . 118

Projektierung von $p_{\mathrm{H}}$-Regelanlagen . . . . . . 344

$p_{\mathrm{H}}$-Wertgeber . . . . . . . . . . . . 297

pneumatisch s. Einzelbegriffe

Potentiometer

Präzisions- . . . . . . . . . . . . . . . . 79

— mit genauer Einstellung . . . . . . . . 258

— schleifer mit steuerbarer Abhebung . . . . . 374

Programmgeber

— mit Wählautomat. . . . . . . . . . . 223

— mit Lochkarten.

Pulsmodulation, Theorie und Praxis der - . . . . . 215

RC-Generator . . . . . . . . . . . . . . 336

Reaktor

Nachbildung des Spalt $-\mathrm{s}$. . . . . . . . . . 21

Siemens- in München. . . . . . . . . . 58

Regelanlagen

Planung von - . . . . . . . . . . . . . . . 18

Wärmetechnische - -5.50 .56$

Regelfläche, Quadratische - . . . 271

Regelpentode . . . . . . . . . . . . . 112

Regelsysteme

s. a. Einzelbegriffe

- mit Laufzeit.

Höhere Ableitung der Regelgröße in $-n$ mit $\dot{S}$ toßstörung . . . . . . . . . . .

Ableitung der Regelgröße in $-\mathrm{n}$ mit statistischer Störung . . . . . . . . . . . . 198 Regelung s. Einzelbegriffe

Regelungsverfahren der Nachrichtentechnik . . . . 214

Regelventile, Bemessung von $-\mathrm{n}$. . . . . . . 351
Regelverstärker, Transistor-Gleichstrom-—. . . . . 330 Regelvorgänge, Darstellung von $-\mathrm{n}$ mittels zeitabhängiger Störungen . . . . . . . . . . . . . . 306 Regler

s. a. Einzelbegriffe

Pneumatischer Einheits- . . . . . . . . . . 20

Elektrischer -

Elektropneumatischer - . . . . . 20, 25

Wärmetechnische —. . . . . . . . . 66

Pneumatische - . . . . . 75, 295, 447

Askania--programm . . . . . . . . . . 142

Universal- und Feld- . . . . . . . . . . . . 144

Norfa-_. . . . . . . . . . 149

Kombinations - mit Istwertanzeige . . . . . 149

Elektronischer - . . . . . . . . . . 189 Relais

- der Nachrichtentechnik . . . . . . . . 214

s. a. Einzelbegriffe

Kontaktschutz- . . . . . . . . . . 22

- Schaltsatz . . . . . . . 40

Maximaldruck - . . . . . . . . . . . 80

Drehspul-Meß_. . . . . . . . . . 112

Drehspul-Klein- . . . . . . . . . . 153

Sauerstoff

Kontinuierliche Messung des in Wasser gelösten $-\mathrm{s} 253$

Phasenaustauschgerät zur Bestimmung des -s-

gehalts. ........... 406

Schreiber

s. a. Einzelbegriffe

Maximum- - . 76

Schweizerische Gesellschaft für Automatik . . . . 59

Spannungsregler mit Elektrometer . . . . . . . . 448 Stabilität

-sgrenze eines Nachlaufreglers mit Stabilisierung 92

Einflu $B$ der Ableitungen auf die - . . . . . 130

Vereinfachte - skriterien für lineare Regelsysteme 170

- von Regelsystemen mit quadratischen Abhän-

gigkeiten im $P I$-Regler. . . . . . . . . . . 240 statistisch

- eines besonderen Regelsystems . . . . . . . . 247

-e Verfahren in der Regelungstechnik . . . . . 11

Ableitung der Regelgröße in Regelkreisen mit -er Störung

Strahlungspyrometer kleiner Ansprechzeit : . . . . . . 226

Stromrichterantriebe. . . . . . . 400

Theorie und Praxis der Regelung von $-\mathrm{n}$. . 5, 44

Strömungsmesser mit magnetischer Anzeige. . . . . 374

Tachometerdynamo, Welligkeit der Gleichspannung von - s und ihr Einfluß auf die Regelung . . . . 203

Tankmessung, Präzisionsgeräte für die - . . . . . . 56

Teleperm-Telepneu-System ..... . . . . . . . 25

Temperatur, Konstante Kälte- - . . . . . . . . . 446

Temperaturmeßumformer . . . . . . . . . 113

Temperaturmessung . . . . . . . . . . . . . . . . 341

Temperaturregler . . . . . . . . . . 300, 413

Pneumatischer - . . . 330

- für Kunststoffverarbeitungsmaschinen. . . 372

Thermoelemente, Miniatur- _. . . . . . . . 436

Thermostat

Präzisions - . . . . . 375

Ultra - .

Titriereinrichtung, Registrierende automatische. . . 408

Toleranzmeßbrücken ........... . 222

Totzeit

Regelkreise mit - . . . . . . . . . . . . . 50

Regelsysteme mit Laufzeit . . . . . . . . . . 89

Beseitigung des Einflusses von - in nichtlinearen

Impulsregelsystemen. . . . . . . . 196

Elektrisches Analogiegerät für - . . . . . . . 266

Optimale Bemessung von Regelsystemen mit - 272,

Transduktor-Ankerregeleinheiten . . . . . . . . 113

Transistor-Netzgerät. . . . . . . . . . . 142

Turbinenregelung, Stetigähnliche - für Kleinwasserkraftanlagen . . . . . . . . . . 2

Übergangscharakteristiken, Näherungsverfahren für aperiodische - . . . . . . . . . . 12 
Übergangsprozesse

Numerische Methode zur Berechnung der — . . 47

Nicholsdiagramm zur Berechnung der Kennwerte einer Übergangsfunktion . . . . . . . . 208 — des Drehstrom-Schleifringläufers . . . . 393, 421

Ventilstellungsregler ...... . . . . . . 452 Verfahrensindustrie, Marktforschung für die Regler in der —. . . . . . . . . 157

Verfahrenstechnik, Pneumatische Multiplikation in der _................ 433

Verhältnisregelung. . . . . . . . . . . 434

Verlusthöhe in Absperr- und Regelorganen . . . . . 193

Verstärker s.a. Einzelbegriffe . . . . . . . . . 448, 450

Vielfachmeßeinrichtung . . . . . . . . . . 39

Wärmeaustauscher, Probleme des Kreuzstrom--s . 438

Wärmeleitfähigkeitsmesser ........ 260
Seita

Wärmemengenregelung. . . . . . . . . . 384

Wärmemengentransmitter .. . . . . . . 114 Werkzeugmaschinen, Elektrische Antriebe, Steuerungen und Regelungen von - . . . . . . . . . . 400

Widerstandsgeber für Druckmessungen. . . . . . . 34

Widerstandsthermometer. . . . . . . . . . . 57

Winkelbeschleunigungsmesser . . . . . . . . . . 37

Wirkdruckwandler, Pneumatischer - . . . 33, 161

Zähler, Elektronischer —. . . . . . . . . . 410

Zählwerk, Pneumatisches — . . . . . . 144

Zeitgeber, Elektronischer — . . . . . . . . 281

Zeitplanregler. . . . . . . . . . . 111

Zeitschaltuhr . . . . . . . . . . . . . 78

Zeitschaltwerk, Präzisions- . . . . 296

Zeitschriftenschau $31-32,69-72,105-108,138-141$, $181-184,217-220,289-292,325-328,364-367$, $401-404,441-444$

Zweipunktregler, Photoelektrischer - . . . . . . . 19

\section{AUTORENVERZEICHNIS}

(Die fetten Zahlen beziehen sich auf die Hauptaufsätze)

\begin{abstract}
Altenhain, F. K. 234
Anke, K. 21

Anders, H. 57, 179

Arnold, K. U. 324

Barth, K. 136

Bauer, A. 92

Bauer, M. 203

Becker, H. 133, 168

Belamin, M. 21

Biederstedt, U. 81

Bopp, K. 230

Bosch, M. 286

Burgholz, R. 178
\end{abstract}

Costake, N. N. 230, 312

Cramer, G. 214

Engel, F. V. A. 287

Engl, W. 67, 165, 324

Euler, K. 89

Ewe, K. 248

Frank, F. 104

Friedemann, H. 399

Früh, K. F. 25, 137

\author{
Gesell, W. 67 \\ Gonnermann, H. 53 \\ Gumin, H. 41
}

Hájek, J. 170

Hannakam, L. 393, 421

Hay, P. 68

Heller, G. 266

Hengstenberg, J. 338

Henning, H. 60

Herschel, R. 196, 216, 240, 362

Hofmann, R. 269, 310

Hutarew, G. 193

Jong, J. J. de 383

Jötten, R. 5, 44

Junker, B. 389

Kaltenecker, H. 30, 317, 324

Kaufmann,H. 216

Kehoe, T. J. 118

Kind, H. 86

Kirschmer, G. 66, 215, 288
Kourim, G, 400

Krägeloh, W. 95

Kretzschmer, F. 351

Kroneberg, D. 117

Kroos, F.-K. 95

Kussl, V. 135

Lichtblau, K. 306

Lintz, H. 160

Luft 256, 399

Lutz, K. 355

Magnus, K. 68,216

Marosky, F. 157

Mesarović, M. 128, 198

Mohr, O. 400

Noebels, H. J. 118

Oldenbourg, R. C. 1

Oppelt, W. 324, 363

Otto, R. 137

Pavlik, E. 433

Peterka, V. 47
Rechberger, H. 2

Richter, H. 280

Schäfer, O. 104, 136

Schink, H. 18

Schlick, K. 66

Schließmann, H. 272, 418

Schlitt, H. 11

Schmid, E. 95

Schraivogel, R. A. 206

Schweigler, W. 136

Silva, M. 118

Starkermann, R. 301

Steudel, H. 103

Strejc, V. 124

Sturm, B. 378

Süss, R. 344

Tschauner, J. 247

Weise, A. 175

Wilmowsky, H. J. v. 427

Wintergerst, E. 160

Zypkin, Ja. S. 196 
